\title{
Das kritisch kranke Kind und seine Angehörigen im Zentrum
}

\author{
Franziska von Arx-Strässlera, Bjarte Rogdo ${ }^{b}$, Thierry Fumeaux ${ }^{c}$, Luca Lavina ${ }^{d}$ \\ a Präsidentin Pflege der SGI, Zürich; ${ }^{b}$ Dr. med., Präsident der Interessengemeinschaft Pädiatrische und Neonatologische Intensivmedizin (IG-PNI) der SGI;
} ${ }^{c}$ Prof. Dr. med., geschäftsführender Präsident der SGI, Nyon; ${ }^{d}$ Generalsekretariat der SGI

Am 4. April 2019 fand im Inselspital Bern das diesjährige Symposium der Schweizerischen Gesellschaft für Intensivmedizin (SGI) statt, welches mittlerweile aus dem Gesellschaftsleben der SGI nicht mehr wegzudenken ist. Zum ersten Mal lag der Fokus dieser Veranstaltung nicht auf der Behandlung von kritisch kranken Erwachsenen, sondern auf Kindern, die auf intensivmedizinische Betreuung angewiesen sind. In mehreren Vorträgen wurden unterschiedlichste Aspekte der neonatologischen und pädiatrischen Intensivmedizin und der Zusammenarbeit mit der Erwachsenenmedizin ausgeleuchtet.

\begin{abstract}
Vierzehn anerkannte neonatologische und pädiatrische Intensivstationen betreuen in der Schweiz kritisch kranke Kinder ab dem Tag ihrer Geburt. Knapp sechs Prozent aller Patientinnen und Patienten, die hierzulande auf Intensivstationen behandelt werden, sind Kinder. Eine der grossen Schwierigkeiten für Kinderintensivstationen und die Kindermedizin im Allgemeinen ist ihre Unterfinanzierung. Da Kinder in der Regel grundversichert sind, ergeben sich nicht genügend Erträge über Zusatzversicherte. Stationär fehlen
\end{abstract}

\section{Eine der grossen Schwierigkeiten für Kinder- intensivstationen und die Kindermedizin im Allgemeinen ist ihre Unterfinanzierung.}

deshalb gemäss Agnes Genewein von Allkids, der Allianz Kinderspitäler der Schweiz, rund fünf Prozent zur Kostendeckung. Auf Dauer führt das dazu, dass Kinderspitäler hinsichtlich ihrer Innovation und Personalentwicklung nicht mehr konkurrenzfähig sind. Eine entsprechende Weiterentwicklung des DRG-Systems soll zu einer Verbesserung der finanziellen Situation beitragen.

Eine weitere Herausforderung, denen Kinderintensivstationen innerhalb und ausserhalb der Schweiz tagtäglich begegnen, liegt in der richtigen Beurteilung der körperlichen und geistigen Entwicklung in den unterschiedlichen Altersstufen eines Kindes. Diese Einschätzung ist für die Einleitung korrekter und altersentsprechender therapeutischer Massnahmen von grosser Bedeutung. So benötigen ein zweijähriges Kleinkind und ein 16-jähriger Jugendlicher etwa völlig unterschiedliche Dosierungen desselben Medikaments. Die exakten Dosierungen müssen zudem für alle kleinen Patientinnen und Patienten zuerst individuell berechnet werden, weil die Medikamente nicht für Kinder, sondern für Erwachsene entwickelt wurden, so Agnes Genewein. Marie-Hélène Perez, Pascaline Fusberti und Manuel Dias vom CHUV zeigen, dass auf Kinder angepasste Lösungen auch beim Spitalmobiliar und bei Geräten häufig Mangelware sind. Beispiele dafür sind Spitalbetten oder Beatmungsgeräte, die wie die meisten Medikamente zum grossen Teil für erwachsene Patientinnen und Patienten entwickelt wurden und deshalb vor der Anwendung für kritisch kranke Kinder modifiziert werden müssen. Doch Not macht bekanntlich erfinderisch! Deshalb

\section{Neue SGI-Broschüre «Die Kinder- intensivstation»}

Am SGI Symposium 2019 wurde die neuste Broschüre der SG vorgestellt, die sich der Kinderintensivstation widmet. Sie gibt Angehörigen wichtige Informationen zur Behandlung und Betreuung von kritisch kranken Kindern. Untermauert werden diese Informationen mit authentischen Erfahrungsberichten betroffener Patienten und Angehöriger, von denen es auf unserer Website noch weitere gibt. Die Publikation ist in vier Sprachen (Deutsch, Französisch, Italienisch und Englisch) verfügbar und kann auf www.sgi-ssmi.ch unter "Broschüren» bestellt werden. 


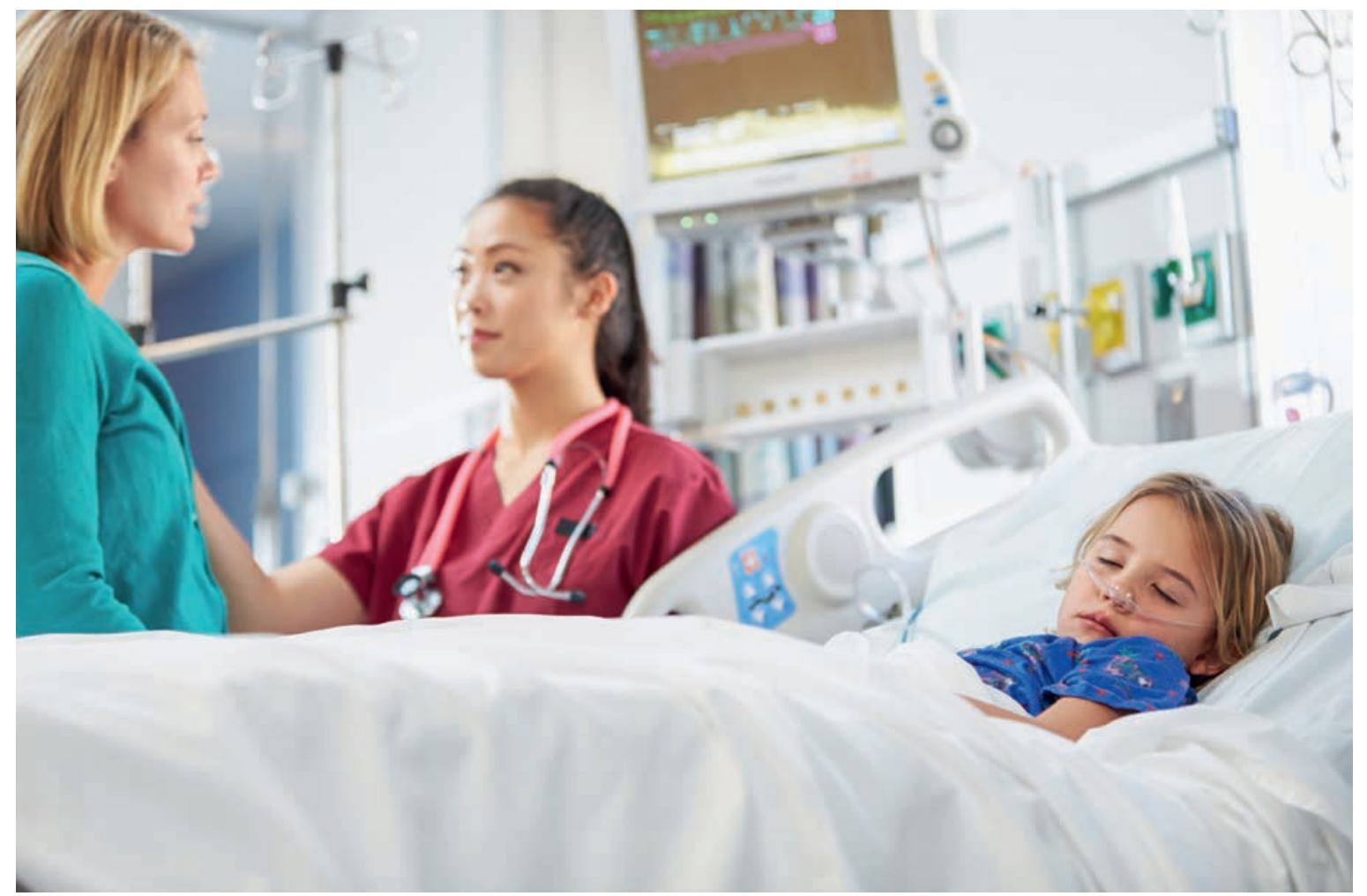

Das Team der Intensivstation begleitet die Familien, nimmt sich Zeit für ihre individuellen Bedürfnisse und gibt umfassend, ehrlich und verständlich Auskunft.

kann die Erwachsenenmedizin von der pädiatrischen und neonatologischen Medizin durchaus etwas lernen, wie Martin Stocker vom Luzerner Kantonsspital erwähnt - etwa in der effizienten Verwendung von Blutproben: So ist sich die Neonatologie heute in der Diagnostik gewohnt, aus so wenig Blut wie möglich ein Maximum an Erkenntnissen über den Gesundheitszustand eines kritisch kranken Neugeborenen zu gewinnen.

\section{Familienzentrierte Begleitung}

Aufgrund des kritischen Gesundheitszustands der Patientinnen und Patienten haben Angehörige auf der Intensivstation stärker als auf anderen Stationen des

\section{Angehörige haben stärker als auf anderen} Stationen des Spitals das Bedürfnis, über alle Behandlungsschritte informiert zu werden.

Spitals das Bedürfnis, möglichst viel Zeit am Krankenbett zu verbringen, über die Therapiemöglichkeiten aufgeklärt und über alle Behandlungsschritte informiert zu werden.

Wenn Familienmitglieder das kritisch kranke Kind zum ersten Mal auf der Intensivstation besuchen, tre- ten sie meist in eine für sie fremde Welt ein und wissen nicht, was sie erwartet. Ausreichend Information ist ein wesentlicher Aspekt, der Angehörigen hilft, die Ohnmacht in der Krise zu überwinden. Das Team der Intensivstation begleitet die Familien, nimmt sich Zeit für ihre individuellen Bedürfnisse und gibt umfassend, ehrlich und verständlich Auskunft. Das Behandlungsteam versucht, dem Kind so viel familiäre Geborgenheit wie möglich zu geben und die Angehörigen gleichzeitig aktiv in den Behandlungsprozess und die pflegerischen Massnahmen einzubeziehen. Diese Involvierung hat zahlreiche Vorteile, wie Vincenzo Cannizzaro vom Universitäts-Kinderspital Zürich betont. Eltern seien, so Cannizzaro, meist jene Personen, die das Kind und seine Krankheitsgeschichte am besten kennen. Zudem seien Eltern heutzutage häufig medizinisch sehr gut informiert, was sie zu kompetenten Partnern in der Entscheidungsfindung macht, wie Ellen Wild vom Ostschweizer Kinderspital in St. Gallen und Sandra Stalder vom Luzerner Kantonsspital in ihrem Symposiumsbeitrag erwähnen. Nur auf einer gegenseitigen Vertrauensbasis gelingt es dem Behandlungsteam und den Angehörigen, wichtige Entscheidungen zu treffen, stellvertretend für das kritisch kranke Kind, das seinen Willen nicht immer selbst äussern kann. 


\section{Von der Kinder- zur Erwachsenenmedizin}

Aufgrund eines angeborenen Herzfehlers hat Janic Spoerri aus Affoltern am Albis seit seiner Geburt viel Zeit auf der Kinderintensivstation verbracht. Zusammen mit seiner Mutter Claudia erzählt er am SGI Symposium aus erster Hand von den Erfahrungen, die er und seine Familie während seiner Kindheit und Jugend gemacht haben. Sie erzählen von den zahlreichen Operationen, denen er sich unterziehen musste, und davon, wie sie den Übergang von der Kinder- zur Erwachsenenmedizin erlebt haben. Der Bericht von Janic und Claudia Spoerri ist auf der SGI-Website als Webcasting zugänglich.

\section{Eine enge Zusammenarbeit zwischen der Kinder- und der Erwachsenenmedizin sowie ein koordinierter Transitionsprozess sind wichtig.}

Wenn aus Kindern Jugendliche werden, übergibt die Pädiatrie Patientinnen und Patienten mit angeborenen Fehlbildungen und chronischen Erkrankungen an die Erwachsenenmedizin, die sich fortan um deren Behandlung kümmert. In der Intensivmedizin liegt dieses Übergangsalter bei circa 16 Jahren, wie Reto Schüpbach vom UniversitätsSpital Zürich in seiner Präsentation bemerkt. In dieser Zeit sind eine enge Zusammenarbeit zwischen der Kinder- und der Erwachsenenmedizin sowie ein koordinierter Transitionsprozess wichtig, sowohl für die Patientinnen und Patienten als auch für die beiden Behandlungsteams. Betroffene Jugendliche, aber auch die Behandlungsteams der Kinder- und der Erwachsenenmedizin können dabei von fixen und vordefinierten Transitionsprogrammen profitieren, wie sie etwa in England in dieser Übergangsphase eingesetzt werden. ${ }^{1}$ Diese Transitionsprogramme bestehen aus mehreren Stufen, in denen sichergestellt wird, dass die Jugendlichen bis zum Abschluss der Transition mit ungefähr 16 Jahren alles Nötige zur eigenen Erkrankung und zu vergangenen und aktuellen Therapien wissen. Denn mit dem Übertritt in die Erwachsenenmedizin werden die Jugendlichen immer selbständiger und übernehmen mehr Verantwortung. Wo früher die Eltern die Ent- scheidungen stellvertretend getroffen haben, werden Informationen den Eltern nur noch in Absprache mit den Jugendlichen mitgeteilt. Dennoch bleibt die Expertise der Eltern für eine erfolgreiche Behandlung wichtig, wie Gaby Stoffel-Gehring vom Kinderspital Zürich und Dina-Maria Jakob vom Inselspital Bern in ihrem gemeinsamen Referat betonen.

Es ist ein zentrales Anliegen der SGI, die Erwachsenenwie auch die Kinderintensivmedizin in ihrer Arbeit gleichermassen $\mathrm{zu}$ vertreten. Die SGI ist deshalb darum bemüht, die Kinderintensivmedizin künftig stärker in ihre Projekte einzubinden. So wird die Kommission für Weiter- und Fortbildung - Ärzte (KWFBÄrzte) in nächster Zeit etwa prüfen, wie gewährleistet werden kann, dass sowohl die Erwachsenen- als auch die Kinderintensivmedizin gleichermassen in der eidgenössischen Facharztprüfung abgebildet sind.

Bildnachweis

(c) Monkey Business Images | Dreamstime.com (Symbolbild)

\section{Das Wichtigste in Kürze}

- Knapp 6\% aller Patientinnen und Patienten auf Schweizer Intensivstationen sind Kinder.

- Die Pädiatrie ist unterfinanziert, stationär fehlen rund fünf Prozent zur Kostendeckung.

- Die Dosierung eines Medikaments muss für jedes Kind individuell berechnet werden, weil die Medikamente und Standarddosierungen für Erwachsene entwickelt werden.

- Angehörige wollen mehr als auf anderen Stationen in das Geschehen eingebunden werden.

- Das Behandlungsteam versucht deshalb, dem Kind so viel familiäre Geborgenheit wie möglich zu geben und die Angehörigen aktiv in alle Massnahmen einzubeziehen.

- Eine koordinierteTransition von der Kinder-zur Erwachsenenmedizin ist wichtig, sowohl für die $\mathrm{Pa}$ tientinnen und Patienten als auch für die Behandlungsteams. 